\title{
Manufacturing and testing of the X-ray collimating mirror for the BEaTriX facility
}

Vecchi, G.; Cotroneo, V.; Ghigo, M.; Basso, S.; Salmaso, B.; Sironi, G.; Spiga, D.; Conconi, P.; Pareschi, G.; Tagliaferri, G.

Total number of authors:

21

Published in:

Proceedings of SPIE

Link to article, DOI:

$10.1117 / 12.2594581$

Publication date:

2021

Document Version

Publisher's PDF, also known as Version of record

Link back to DTU Orbit

Citation (APA):

Vecchi, G., Cotroneo, V., Ghigo, M., Basso, S., Salmaso, B., Sironi, G., Spiga, D., Conconi, P., Pareschi, G., Tagliaferri, G., Burwitz, V., Hartner, G., Müller, T., Rukdee, S., Schmidt, T., Christensen, F., Ferreira, D. D. M., Gellert, N., Massahi, S., ... Ferreira, I. (2021). Manufacturing and testing of the X-ray collimating mirror for the BEaTriX facility. In S. L. O'Dell, J. A. Gaskin, \& G. Pareschi (Eds.), Proceedings of SPIE: Optics for EUV, X-Ray, and Gamma-Ray Astronomy X [118220N] SPIE - International Society for Optical Engineering. Proceedings of SPIE - The International Society for Optical Engineering Vol. 11822 https://doi.org/10.1117/12.2594581

\section{General rights}

Copyright and moral rights for the publications made accessible in the public portal are retained by the authors and/or other copyright owners and it is a condition of accessing publications that users recognise and abide by the legal requirements associated with these rights.

- Users may download and print one copy of any publication from the public portal for the purpose of private study or research.

- You may not further distribute the material or use it for any profit-making activity or commercial gain

- You may freely distribute the URL identifying the publication in the public portal 


\title{
Manufacturing and testing of the X-ray collimating mirror for the BEaTriX facility
}

\author{
G. Vecchi ${ }^{\text {1a }}$, V. Cotroneo ${ }^{\mathrm{a}}$, M. Ghigo ${ }^{\mathrm{a}}$, S. Basso ${ }^{\mathrm{a}}$, B. Salmaso ${ }^{\mathrm{a}}$, G. Sironi ${ }^{\mathrm{a}}$, D. Spiga ${ }^{\mathrm{a}}$, P. Conconia ${ }^{\mathrm{a}}$, \\ G. Pareschi ${ }^{\mathrm{a}}$, G. Tagliaferri ${ }^{\mathrm{a}}$, V. Burwitz ${ }^{\mathrm{b}}$, G. Hartner ${ }^{\mathrm{b}}$, T. Müller ${ }^{\mathrm{b}}$, S. Rukdee ${ }^{\mathrm{b}}$, T. Schmidt ${ }^{\mathrm{b}}$, F. \\ Christensen $^{\mathrm{c}}$, D. D. M. Ferreira ${ }^{\mathrm{c}}$, N. C. Gellert ${ }^{\mathrm{c}}$, S. Massahi ${ }^{\mathrm{c}}$, M. Bavdaz $^{\mathrm{d}}$, I. Ferreira ${ }^{\mathrm{d}}$

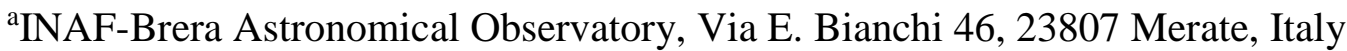 \\ ${ }^{b}$ MPI für extraterrestrische Physik, Giessenbachstr., 85748 Garching, Germany \\ ${ }^{c}$ DTU Space, Technical University of Denmark, Elektrovej 327, 2800 Kgs. Lyngby, Denmark \\ ${ }^{\mathrm{d}}$ European Space Agency, ESTEC, Keplerlaan 1, 2200 AG Noordwijk, The Netherlands
}

\begin{abstract}
The BEaTriX (Beam Expander Testing X-ray) facility under construction at INAF-Brera Astronomical Observatory aims at performing the acceptance tests of the Silicon Pore Optics mirror modules of the ATHENA (Advanced Telescope for High-ENergy Astrophysics) X-ray observatory. The facility implements a grazing-incidence collimating mirror that, together with a monochromator and a beam expander stages based on crystals, enables the full X-ray illumination of the mirror modules under test. We present the development and test of the collimating mirror, a paraboloid sector having an optical surface of $400 \mathrm{~mm} \times 60 \mathrm{~mm}$ and sagittal radii of about $155 \mathrm{~mm}$. The ground and lapped optics made of HOQ 310 fused quartz was corrected by bonnet polishing. In this paper, we report on the smoothing of mid-to-high spatial frequency error by pitch-tool polishing process, and on the correction of residual surface shape errors by ion-beam figuring process, both performed at INAF-Brera Astronomical Observatory. We present the X-ray test campaigns carried out on the mirror at PANTER facility, before and after coating it with a Pt layer via magnetron sputtering at DTU Space. The results provide an overview of the mirror performance in terms of angular resolution pre- and post-coating deposition.
\end{abstract}

Keywords: ATHENA, BEaTriX, X-ray test facility, pitch polishing, ion beam figuring, X-ray collimator, PANTER, coating

\section{INTRODUCTION}

ATHENA (Advanced Telescope for High-ENergy Astrophysics) is the second Large Class ESA mission [1], with launch foreseen in the early 2030s, aiming at studying the hot and energetic Universe in the X-ray band. The ATHENA X-ray telescope, with $12 \mathrm{~m}$ focal length and angular resolution of $5 \operatorname{arcsec}$ Half-Energy Width (HEW) at $1 \mathrm{keV}$ is based on the Silicon Pore Optics (SPO) modular technology [2]. The large X-ray mirror assembly of $\sim 2.5 \mathrm{~m}$ diameter is composed of more than 600 co-aligned SPO modules, providing an effective area of $1.4 \mathrm{~m}^{2}$ at $1 \mathrm{keV}$ [3]. Each X-ray Optical Unit (XOU) is made of 35 processed silicon plates, while four XOUs are aligned and integrated in the SPO Mirror Module (MM) using synchrotron radiation [4]. X-ray testing of MMs are currently done at XPBF 2.0 [5] and PANTER [6] facilities, while a new X-ray beamline for the assembly and characterization of MMs is in construction at ALBA synchrotron [7]. The BEaTriX (Beam Expander Testing X-ray) facility is a new type of beamline for mirror acceptance testing and calibration, under realization at INAF-Brera Astronomical Observatory [8], and funded by ESA, AHEAD, ASI and INAF. BEaTriX was designed to provide a broad $(170 \mathrm{~mm} \times 60 \mathrm{~mm})$ and uniform X-ray beam with 1.5 arcsec HEW residual divergence, at either $1.49 \mathrm{keV}$ or $4.51 \mathrm{keV}$ energy $[9,10]$. The BEaTriX facility aims at performing the acceptance test (Point Spread Function, PSF, and effective area) of the ATHENA SPO MM's at the fast rate of $3 \mathrm{MM} /$ day. In the optical design of BEaTriX, the X-ray source is at the focus of a grazing incidence $(\sim 0.9 \mathrm{deg})$ paraboloidal mirror. The collimated beam exiting the mirror with a $4 \mathrm{~mm} \times 60 \mathrm{~mm}$ section is spectrally filtered by symmetrically cut silicon crystals and expanded in the horizontal direction by an asymmetrically cut silicon crystal [11].

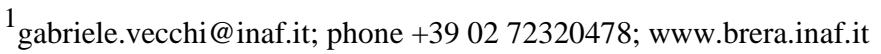

Optics for EUV, X-Ray, and Gamma-Ray Astronomy X, edited by Stephen L. O'Dell,

Jessica A. Gaskin, Giovanni Pareschi, Proc. of SPIE Vol. 11822, 118220N · ()

2021 SPIE $\cdot$ CCC code: 0277-786X/21/\$21 - doi: 10.1117/12.2594581 
The final beam of size $170 \mathrm{~mm} \times 60 \mathrm{~mm}$ enables full illumination of SPO MMs. Fig. 1 shows the BEaTriX optical layout.

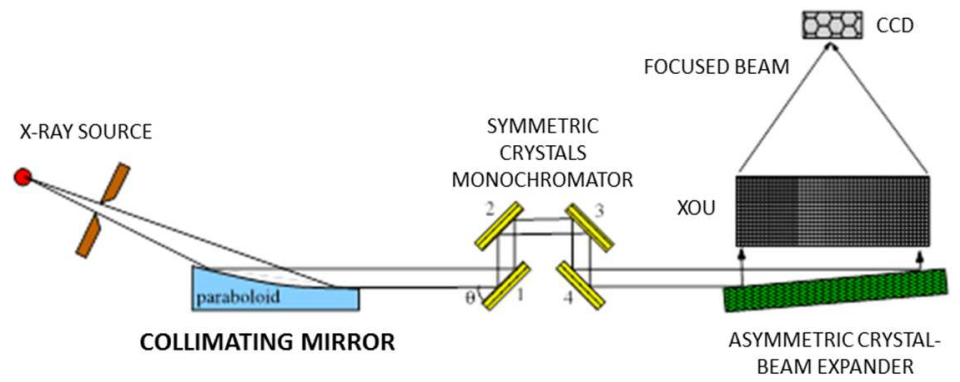

Figure 1. Optical layout of the BEaTriX facility [12].

This paper focuses on the manufacturing and testing of the paraboloidal collimating X-ray mirror. Section 2 briefly reports some recent analysis on manufacturing tolerances, that allowed us to somehow relax the surface roughness requirement. We present and discuss the mirror manufacturing, including the polishing, figuring and measurement analysis done at INAF- Brera Astronomical Observatory. Section 3 presents the X-ray test campaigns performed at the PANTER facility in order to assess the mirror performance. We therein give a performance overview in angular resolution achieved by the mirror pre- and post-coating, comparing measurements and simulations of the best focus PSF. The platinum coating, needed to endow the mirror surface with reflective properties at $4.51 \mathrm{keV}$, was deposited at DTU Space.

\section{MANUFACTURING THE COLLIMATING MIRROR}

We hereafter describe the mirror requirements, the procurement and the first step of manufacturing which employed the bonnet polishing technique we described elsewhere [13]. In this paper, we present the subsequent manufacturing steps we developed, based on the pitch-tool polishing and the ion-beam figuring techniques.

\subsection{Manufacturing tolerances}

We computed the largest figure and roughness errors compatible with a mirror of 0.5 arcsec HEW at $4.51 \mathrm{keV}$ by means of the Wavefront propagatIon Simulation codE (WISE) code [14]. We generated the figure and roughness longitudinal profiles from mono-dimensional Power Spectral Density (PSD) modeled by broken power laws, where the boundary between the figure profile and roughness is arbitrarily set at $1 \mathrm{~mm}$. New simulations were performed to check if the initial requirement on roughness ( $<3.5 \AA$ Root Mean Square, RMS, error within $1 \mathrm{~mm}-1 \mu \mathrm{m}$ length range) could be relaxed to some extent. Table 1 shows the HEW values associated to the original PSD of reference (case 1) and to new PSDs, which mitigate the roughness requirement progressively (case 2-3-4-5). The analysis shows we can raise the requirement on roughness from $3.5 \AA$ to $\sim 7 \AA$ without significantly degrading the corresponding HEW value up to 4.51 $\mathrm{keV}$.

Table 1. Estimated HEW and RMS values in various length range for different PSD cases.

\begin{tabular}{|c|c|c|c|c|c|c|c|c|c|}
\hline & \multicolumn{2}{|c|}{ profile } & \multicolumn{2}{|c|}{ roughness } & \multirow{2}{*}{$\begin{array}{c}\text { HEW } \\
{[\operatorname{arcsec}]}\end{array}$} & \multirow{2}{*}{$\begin{array}{c}\mathrm{rms}_{\text {from PSD }}[\AA] \\
(436 \mathrm{~mm} \div 10 \\
\mu \mathrm{m})\end{array}$} & \multirow{2}{*}{$\begin{array}{l}\mathrm{rms}_{\text {from PSD }}[\AA] \\
(436 \mathrm{~mm} \div 15 \\
\mathrm{mm})\end{array}$} & \multirow{2}{*}{$\begin{array}{c}\left.\mathrm{rms}_{\text {from PSD }}[\AA]\right] \\
(15 \mathrm{~mm} \div 10 \mu \mathrm{m})\end{array}$} & \multirow{2}{*}{$\begin{array}{c}\mathrm{rms}_{\text {from PSD }}[\AA \AA \\
(5 \mathrm{~mm} \div 10 \mu \mathrm{m}) \\
/ \\
(1 \mathrm{~mm} \div 1 \mu \mathrm{m})\end{array}$} \\
\hline & $\mathrm{n}$ & $\mathrm{K}_{\mathrm{n}}$ & $\mathrm{n}$ & $\mathrm{K}_{\mathrm{n}}$ & & & & & \\
\hline 1 & 2.5 & $5 \times 10^{-4}$ & 1.0 & 18 & 0.50 & 122.8 & 122.5 & 8.1 & $4.3 / 3.5$ \\
\hline 2 & 2.5 & $5 \times 10^{-4}$ & 1.0 & 20 & 0.50 & 122.8 & 122.5 & 8.16 & $4.4 / 3.7$ \\
\hline 3 & 2.5 & $5 \times 10^{-4}$ & 0.85 & 50 & 0.49 & 122.8 & 122.5 & 8.3 & $4.7 / 4.6$ \\
\hline 4 & 2.5 & $5 \times 10^{-4}$ & 0.75 & 100 & 0.50 & 122.8 & 122.5 & 8.5 & $5.1 / 5.7$ \\
\hline 5 & 2.5 & $5 \times 10^{-4}$ & 0.66 & 200 & 0.52 & 122.8 & 122.5 & 8.9 & $5.6 / 7.3$ \\
\hline
\end{tabular}



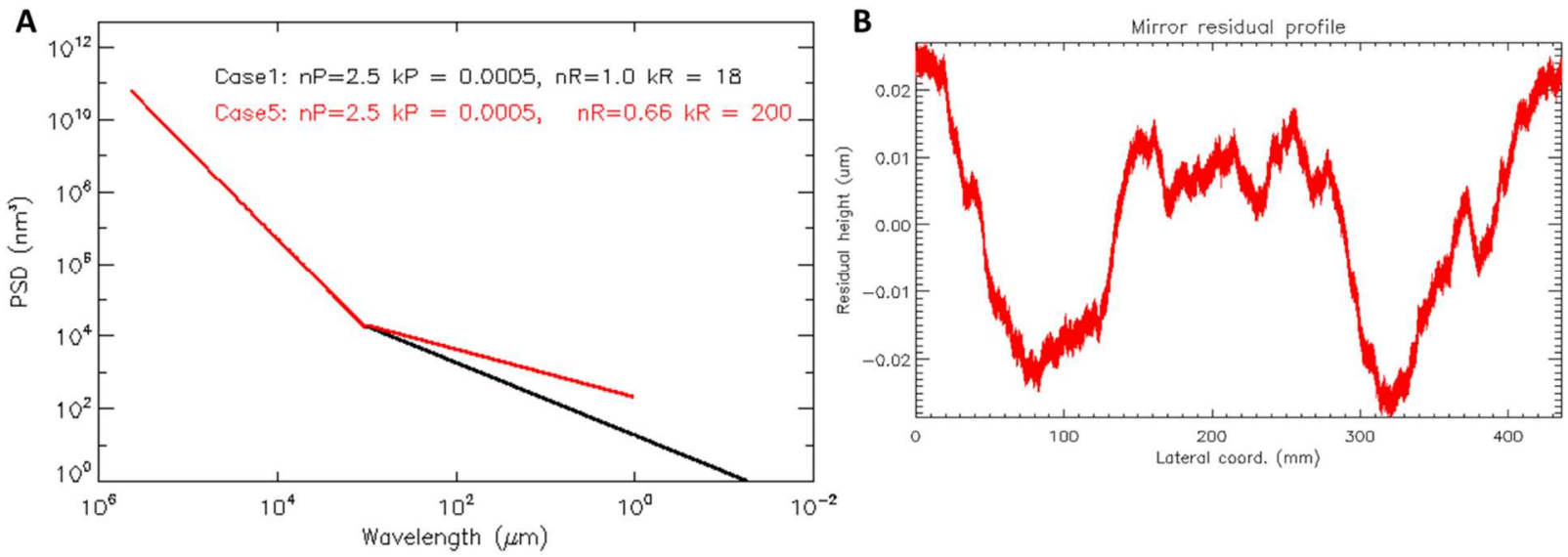

Figure 2. A. Original reference PSD (black, case 1 of Table 1) and new reference PSD (red, case 5 of Table 1) with relaxed tolerance on roughness value. B. One of the infinitely possible profiles consistent with the new reference PSD.

Fig. 2A compares the previous (black, case 1 of Table 1) to the new reference PSD (red, case 5 of Table 1) which represents the actual tolerance on the roughness value, close to $7 \AA$. Achieving this roughness value at spatial wavelengths below $1 \mathrm{~mm}$ is the purpose of pitch polishing process, described in the next subsection. Fig. 2B shows a possible longitudinal profile generated pseudo-randomly from the new reference PSD.

It is worth to mention that in the first version of the optical design [10], we had estimated that a maximum tolerable HEW of 3 arcsec was to be reached through the manufacturing process. Afterwards, with the purpose to counterbalance some adjustments of the overall error budget, we turned the manufacturing tolerances stricter, so as to leave the target of divergence tolerance of the BEaTriX beam within 1.5 arcsec. This turned out into a very tight requirement of 0.5 arcsec HEW for the surface quality of the mirror [13]. We compare the two requirements in Fig. 3, where we plot the reference PSDs yielding 0.5 and 3 arcsec HEW at $4.51 \mathrm{keV}$. The same two reference PSDs will be presented in subsection 2.4 and 2.5, compared with the surface metrology PSDs. The short wavelength $(<1 \mathrm{~mm})$ regime, characterizing the roughness contribution, is unchanged in the two reference PSDs, and it corresponds to the one of Fig. $2 \mathrm{~A}$ (displayed in red) with relaxed tolerance.

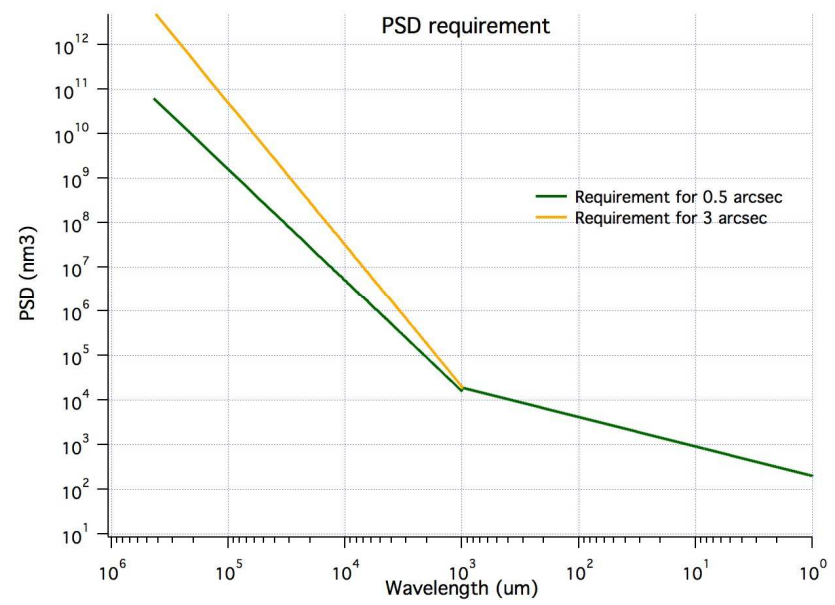

Figure 3. Reference PSD for 0.5 arcsec HEW (green line) compared to 3 arcsec HEW requirement (orange line). 


\subsection{Pitch smoothing}

Fig. 4A is a drawing of the workpiece, Fig. 4B shows the HOQ 310 fused quartz blank, which was ground and preliminarily lapped at Carl Zeiss SMT GmbH. The size of the workpiece is $456 \mathrm{~mm} \times 100 \mathrm{~mm} \times 50 \mathrm{~mm}$ and the ground surface area was $456 \mathrm{~mm} \times 80 \mathrm{~mm}$. The optical surface, initially set to $436 \mathrm{~mm} \times 60 \mathrm{~mm}$ area, is represented in Fig. $4 \mathrm{C}$ by the black-dotted area. The red area is the projection of the beam expander $(170 \mathrm{~mm} \times 60 \mathrm{~mm})$ back traced to the mirror or, in other words, the area of the paraboloid involved in the generation of the expanded beam. The smallest rectangular area bordering the red one has $400 \mathrm{~mm} \times 60 \mathrm{~mm}$ area and it was assumed as the new optical surface at the center of the workpiece.
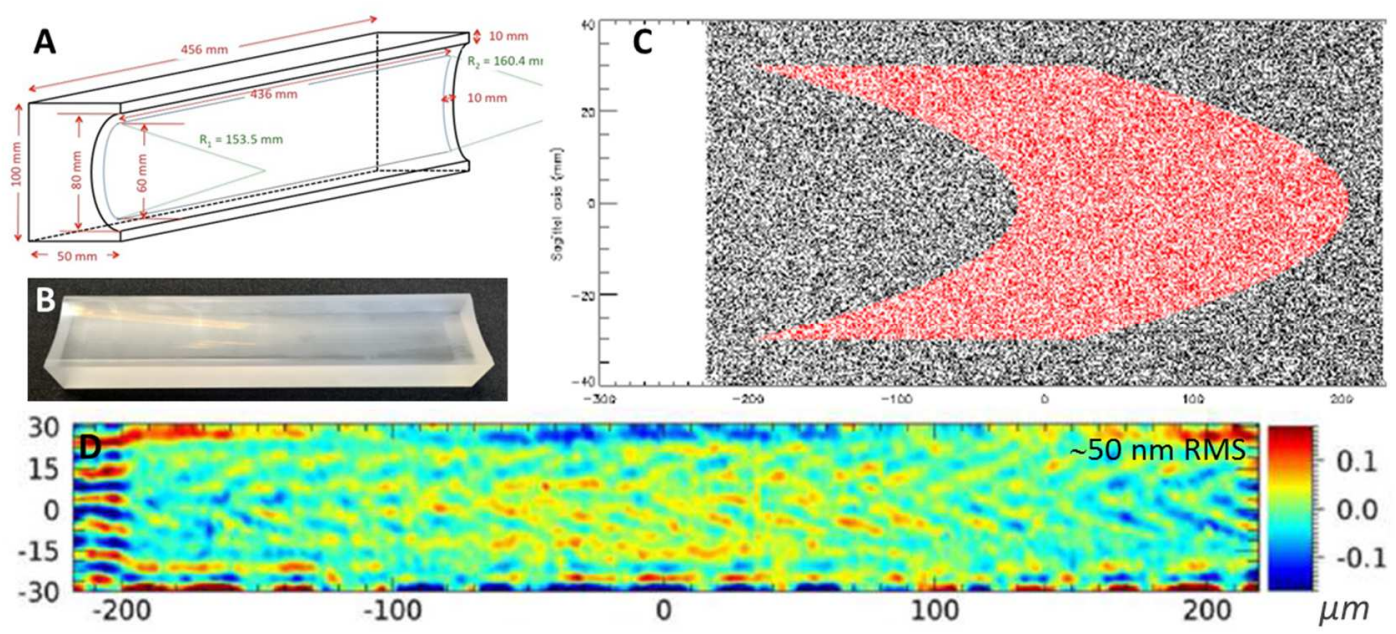

Figure 4. A. Drawing of the workpiece. B. Mirror blank manufactured at Carl Zeiss SMT GmbH. C. Projection area (red area) of the BEaTriX beam expander back to the paraboloidal mirror surface area. D. Longitudinal error map after bonnet polishing [13].

Fig. 4D shows the longitudinal error map measured with optical profilometry after bonnet polishing [13]. We continued the surface improvement by implementing a new, ad hoc, pitch-based tool, designed to fit the paraboloid surface and to smooth the waviness error shorter than $\sim 7 \mathrm{~mm}$. We assumed this value to mark the onset of spatial length range not or poorly correctable by bonnet polishing technique. The oscillation amplitude of the pitch tool across the optical surface was defined accordingly. We planned to run pitch-based process employing the Zeeko IRP1200 machine at INAF-Brera Astronomical Observatory, shown in Fig. 5A. This allowed us to take advantage of the thermal stability and cleanliness of the host ISO7 room.
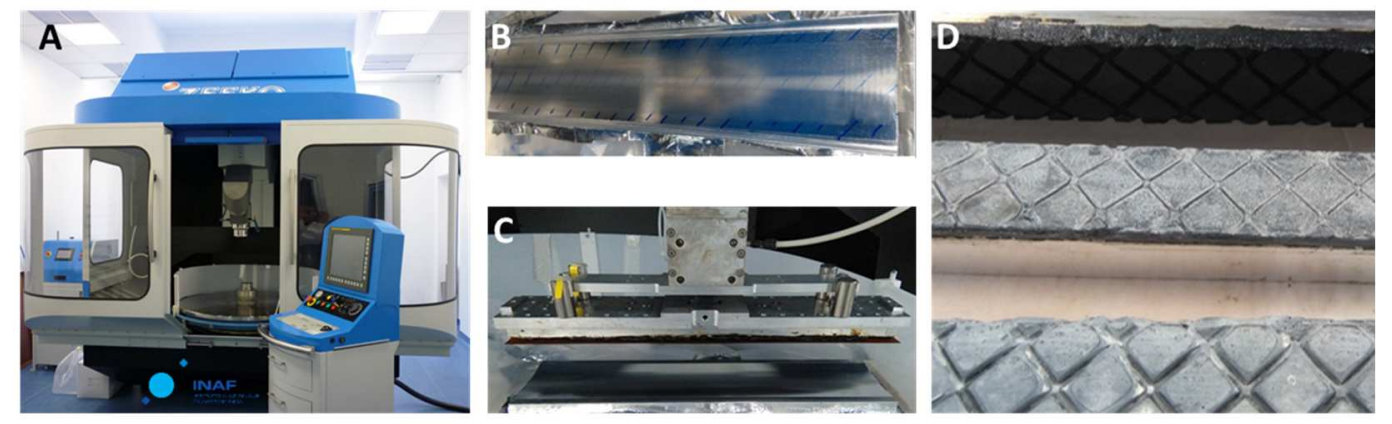

Figure 5. A. Photo of the Zeeko IRP1200 machine installed at INAF-Brera Astronomical Observatory. B. Aluminum replica of the mirror workpiece. C. Pitch tool lifted above the replica workpiece during preliminary test activity. D. Different pitch pads fabricated for the smoothing process development.

The tool was designed in order to fit into the robotic arm of the IRP1200 machine in place of the bonnet. The tool holds the pitch pad by means of an air-bearing carriage. The applied weight force was limited by the maximum bearable load by the machine interface, and was adjusted by accommodating a number of tungsten posts to control the pressure 
applied at the pitch-glass interface. The tool positioning and motion was controlled by the 7-axis CNC system of the machine. Preliminary polishing test were set and performed on a dummy part, an aluminum replica workpiece, shown in Fig. 5B, fabricated on purpose. Fig. 5C shows the pitch tool lifted above the dummy part. Pads were prepared using pitches of different hardness, with length close to that of the workpiece. Fig. 5D shows three different pads, i.e., one not yet used, made of pristine black pitch and two, which were worn by usage, whitish because charged with cerium-oxide abrasive particles contained in the Opaline-based slurry.

\subsection{Ion-beam figuring}

We used the technique of ion-beam figuring (hereafter IBF) to correct the shape error left on the surface after pitch polishing. In particular, we addressed the longitudinal profile errors and the azimuthal radii of curvature to meet the paraboloid design and focal length requirements. Fig. 6A shows the IBF facility developed at INAF-Brera Astronomical Observatory [15]. The IBF technique removes material from an optic surface by the kinetic impact provided by a beam of Argon ions.
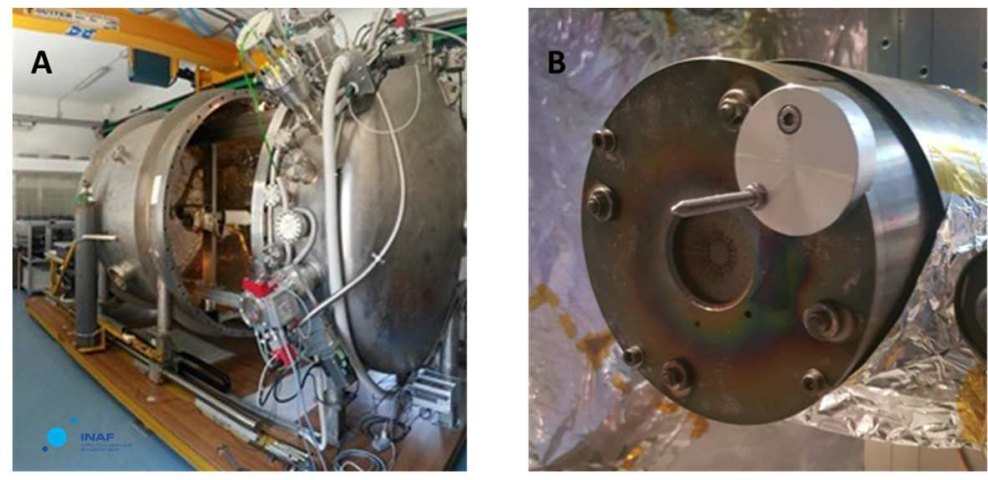

Figure 6. A. The IBF facility developed and installed at INAF-Brera Astronomical Observatory. B. Close view of the ion source head equipped with $15 \mathrm{~mm}$ graphite grid set and metal tip for alignment purpose.

The process occurs in a vacuum chamber equipped with a mounting frame $(1.7 \mathrm{~m} \times 1.4 \mathrm{~m})$ which defines the lateral full scanning area of the source. The third travel axis allows the source to follow the sag of curved optics, keeping a constant working distance, in this case set to $70 \mathrm{~mm}$. Our IBF employs a hollow cathode DC Kaufmann ion source type. The water-cooled head source shown in Fig. 6B mounted a $15 \mathrm{~mm}$ focused grid set made in pyrolytic graphite, delivering a beam of Full Width Half Maximum (FWHM) varying in the range 10-15 mm, depending on the grid-optics distance and on setting parameters of the source. We performed the calibration of the removal rate on fused silica planar samples (Fig. 7A). By combining IBF cycles in stationary conditions and the interferometric characterization of the substrate surface shape we derived the removal function associated to the specific process applied. This function (an example of it is shown in Fig. 7B), together with the surface error map, is the required input to calculate the correction time matrix. Fig. 7C is a picture of the collimating mirror mounted inside the IBF chamber during one process cycle.
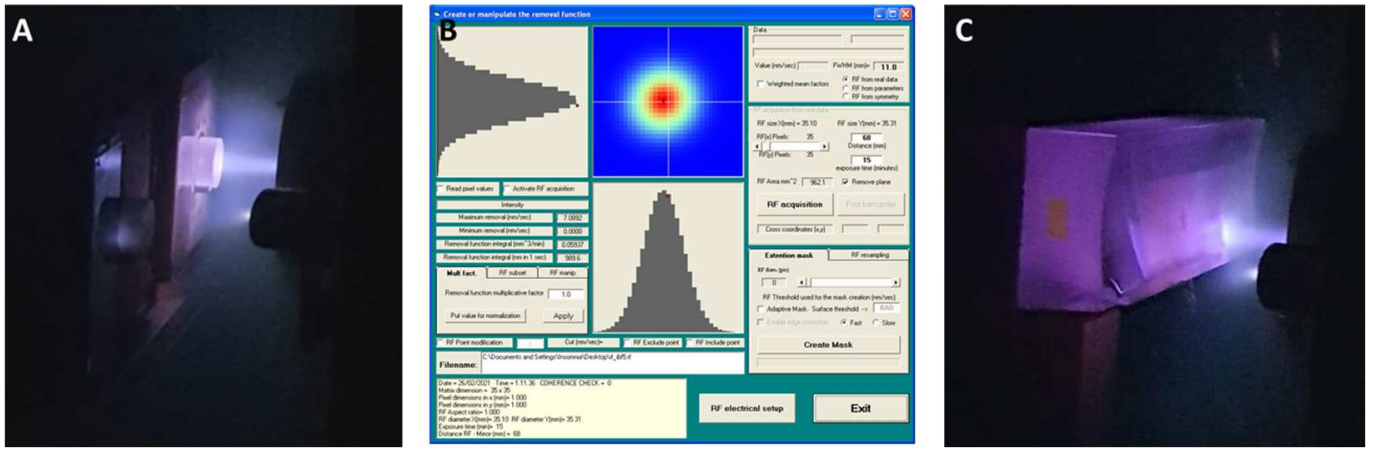

Figure 7. A. IBF process on planar reference samples. B. Example of a removal function set in the dialog box of the analysis software. C. The collimating mirror undergoing one IBF cycle. 


\subsection{Metrology: surface topography}

We measured the mirror with the CCI (Coherence Correlation Interferometer, by AMETEK Taylor Hobson) interferometer at Media Lario S.r.l. to check the topography evolution of the optical surface throughout the manufacturing process. By measuring the surface topography by three objectives $(2.5 \mathrm{X}, 10 \mathrm{X}, 50 \mathrm{X})$ with different Field Of View (FOV), we evaluated the roughness over a wavelength range from a few millimeters $\left(2.5 \mathrm{X}\right.$ obj., $1 \times 6 \mathrm{~mm}^{2}$ FOV) down to few micrometers (50X obj., $\left.0.3 \times 0.3 \mathrm{~mm}^{2} \mathrm{FOV}\right)$. The measurements performed with CCI $2.5 \mathrm{X}$ objective were important to assess the longitudinal waviness error reduction by the pitch smoothing process around 1 $\mathrm{mm}$. This (mid-frequency) range is critical for both manufacturing and metrology. The deterministic techniques as bonnet polishing and IBF based on compliant or contactless tools are not effective in this range. The characterization of the surface in the $\mathrm{mm}$ error wavelength region is challenging, being intermediate for instruments optimized either for (high frequency) roughness or for (low frequency) surface figure measurements. Fig. 8 shows two topography maps measured with 50X (panel A) and 10X (panel B) magnifications after the last run of manufacturing. The RMS values are representative of the surface quality achieved. We computed the RMS values along the two axes of the squared map separately. The horizontal axis "Az" stands for the azimuthal (sagittal) direction, whereas the vertical axis "Lo" indicates the longitudinal (tangential or meridional) direction on the paraboloid surface. The optical surface was sampled at nine different positions with each objective, three along the central longitudinal axis ( 0 deg azimuth), whereas the six remaining were taken at \pm 9 deg symmetric azimuthal positions, nearing the edge of the optical surface area. We repeated the measurements at the same nine nominal positions at each CCI measurement series, regardless of positioning accuracy. Fig. 9C shows the $\mathrm{CCI}$ aligned in front of the mirror.
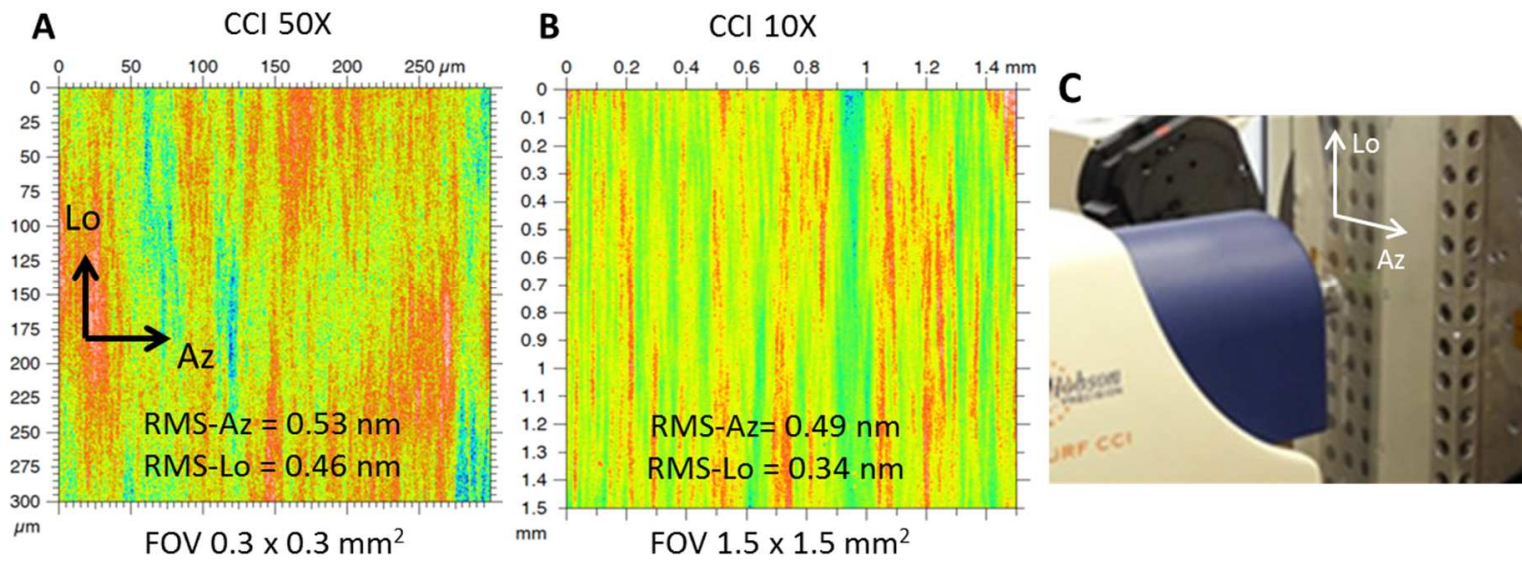

Figure 8. A. Topography map taken with CCI 50X (pixel size $\sim 0.6 \mu \mathrm{m}$ ). The longitudinal (Lo) and azimuthal (Az) axes are displayed. RMS values were calculated separately along the axes. B. Topography map taken with CCI 10X (pixel size $\sim 2.9$ $\mu \mathrm{m})$. C. Photograph of the CCI instrument at Media Lario S.r.l.

We did not include CCI 2.5X azimuthal data to compute the corresponding PSD, as the maps are aberrated by the curvature of the mirror. Fig. 9A shows the PSD calculated from azimuthal profile data measured with 10X and 50X objective and stitched together. Fig. 9B shows the PSD computed from the longitudinal profile data from the full collection of three objectives. 

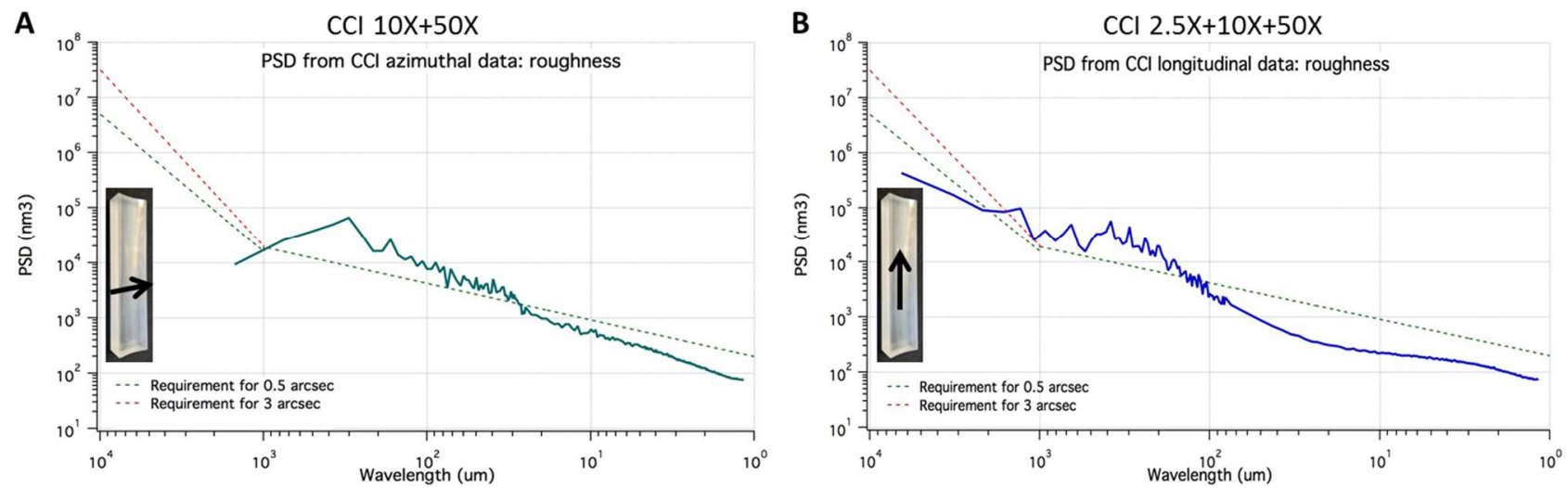

Figure 9. A. Mono-dimensional roughness PSD inferred from the CCI (10X and 50X objectives) azimuthal profile data. B. Mono-dimensional roughness PSD from CCI (2.5X, 10X and 50X objectives) longitudinal profile data. In both panels, the reference PSDs for 0.5 and 3 arcsec HEW mirror quality are represented.

\subsection{Metrology: optical profilometry}

The longitudinal profile errors were measured during sessions of optical profilometry [16] performed at Media Lario S.r.l. throughout the manufacturing process. The radii of curvature in the azimuthal direction were measured with a 3D Coordinate Measuring Machine (CMM) at INAF-Brera Astronomical Observatory. After the pitch-based process used to improve the mid-to-high spatial frequency errors, the low frequency errors, introduced by the process itself, were corrected with IBF. Fig. 10 shows the longitudinal error map measured at the end of the manufacturing.

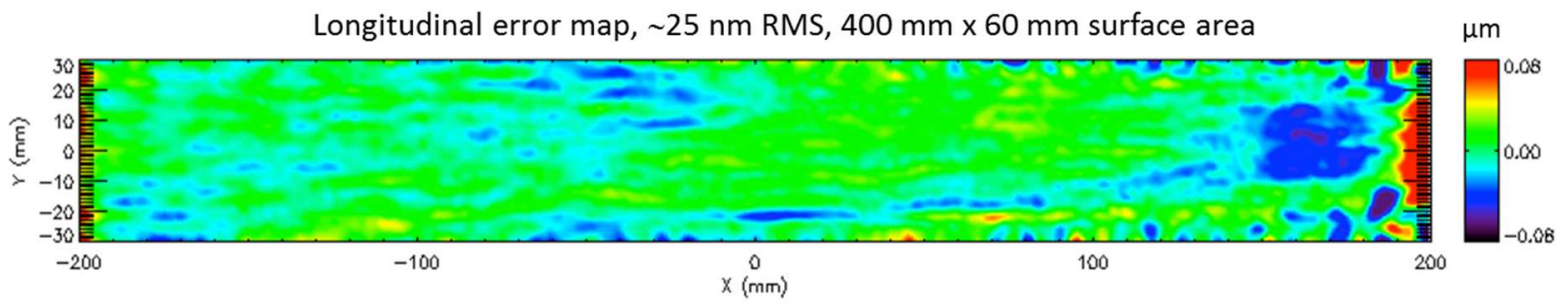

Figure 10. Longitudinal error map after the manufacturing process. The RMS value is $\sim 25 \mathrm{~nm}$ over the $400 \mathrm{~mm} \times 60 \mathrm{~mm}$ optical area.

Fig. 11 shows the PSDs of the longitudinal residual errors in the $2-400 \mathrm{~mm}$ range measured before IBF and after final pitch smoothing and IBF. The final PSD meets the 3 arcsec reference PSD in the long wavelength side of the spectrum. Moving towards the $\mathrm{mm}$ range, the same PSD, inferred from profilometry data, appears increasingly noisy and remains above the reference PSDs. In the $\mathrm{mm}$ range, we assumed the PSD inferred from CCI data shown in Fig 9B as the more accurate description of the residual error. 


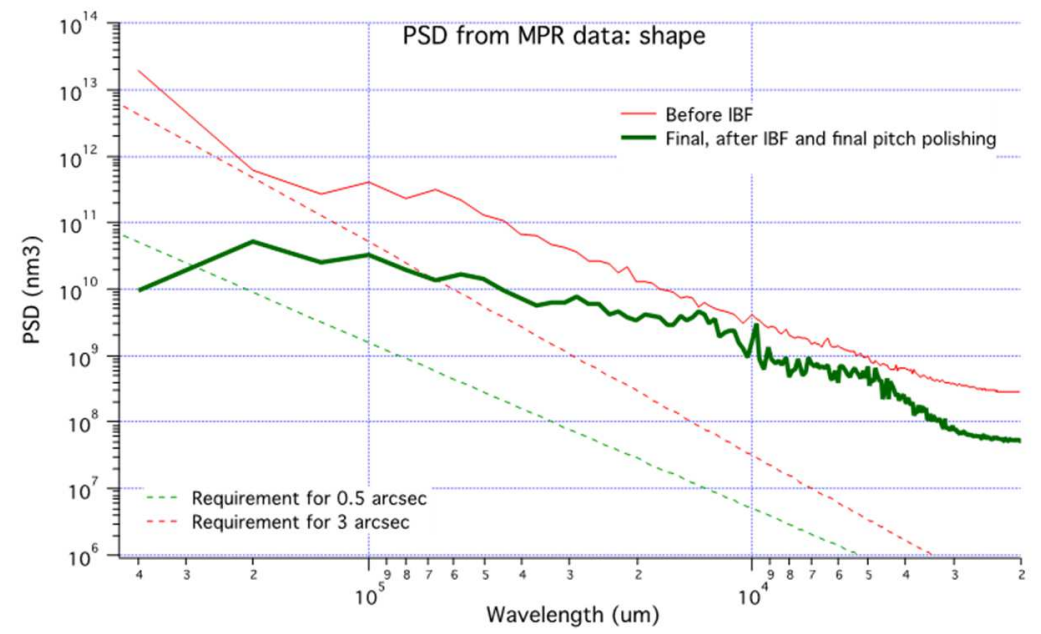

Figure 11. PSDs calculated from the longitudinal error maps measured with the optical profilometer (MPR) at Media Lario S.r.l. The reference PSDs for 0.5 and 3 arcsec HEW mirror quality are represented.

\section{X-RAY TESTING}

\subsection{Uncoated mirror}

The mirror was installed in the PANTER vacuum chamber mounted on the kinematic support previously assembled and aligned at INAF-Brera Astronomical Observatory and used for optical metrology. This configuration is visible in Fig. 12A. In front of the support, on the entrance side of the beam, there was a slit defining the illuminated rectangular area (400 $\mathrm{mm} \times 60 \mathrm{~mm}$ on mirror surface) to fit the optical surface area. Therefore, the surface area set for X-ray test was larger than the red-dotted area in Fig. 4C. The slit was made of a stainless steel $0.5 \mathrm{~mm}$ thick slab, enough to stop Xrays at $4.51 \mathrm{keV}$. Fig. 12B is a picture of the mirror mounted on the support with the illumination slit.
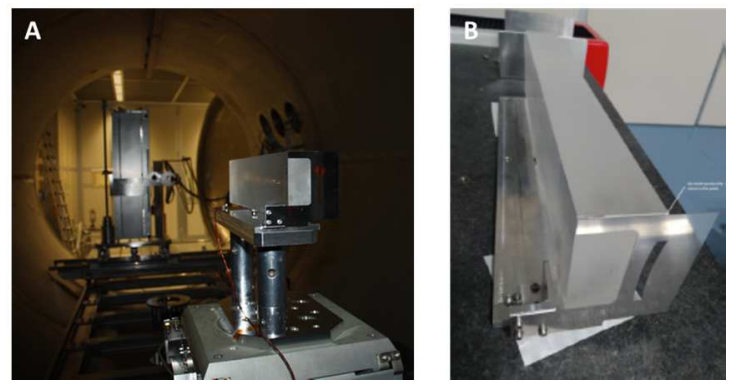

Figure 12. A. Photo of the mirror mounted in the PANTER vacuum chamber, viewed from the side of incoming beam. B. The slit to define the illumination area was mounted and aligned onto the mirror with CMM at INAF-Brera Astronomical Observatory.

All the measurements on the uncoated mirror were made at $1.49 \mathrm{keV}$, using both the TRoPIC (75 $\mu \mathrm{m}$ pixel, corresponding to $\sim 3.3 \mathrm{arcsec}$ ) and PIXI ( $20 \mu \mathrm{m}$ pixel, corresponding to $\sim 0.87$ arcsec) detectors. Fig. 13A shows the best focus PSF measured using the diverging beam setup and TRoPIC detector. The angular resolution in terms of HEW was measured to 4.8 arcsec, in perfect agreement with the simulated 5.0 arcsec, inferred from metrological data (Fig. 13B). 

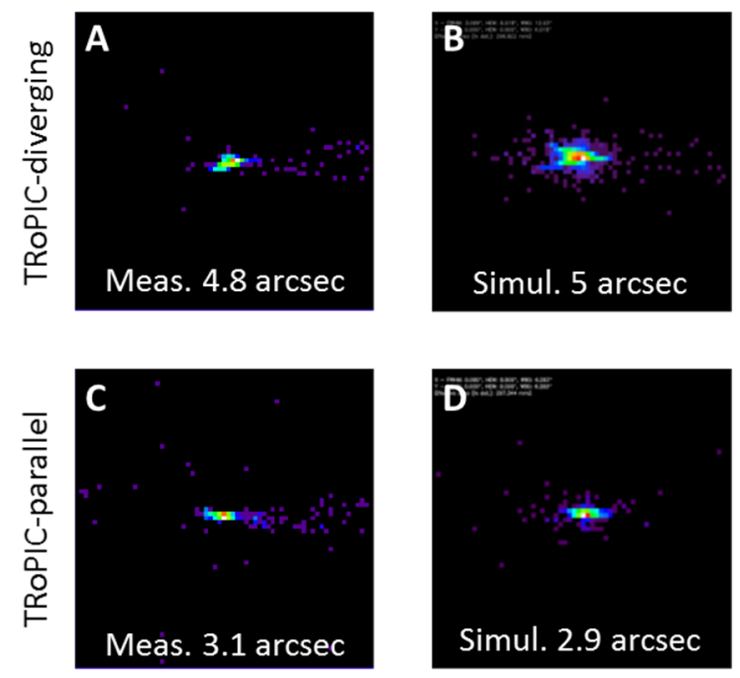

Figure 13. Panel A (C). Best focus measured with TRoPIC in diverging (parallel) beam setup, HEW $=4.8$ arcsec (3.1 $\operatorname{arcsec}$ ). Panel B (D). Theoretically predicted best focus PSF corresponding to A (C) configurations, respectively. The simulated HEW values are displayed. Logarithmic color scale. The images have $5 \mathrm{~mm}$ size. Measurements were made at $1.49 \mathrm{keV}$.

Due to the finite distance of the PANTER source, a beam divergence of $1.66 \mathrm{arcsec} / \mathrm{mm}$ with respect to the central axis was assumed. In order to reduce the aberration of the image due to the beam divergence, the Fresnel zone plate available at PANTER and operating at $1.49 \mathrm{keV} \mathrm{[17]} \mathrm{was} \mathrm{inserted} \mathrm{and} \mathrm{aligned} \mathrm{with} \mathrm{respect} \mathrm{to} \mathrm{the} \mathrm{mirror.} \mathrm{The} \mathrm{measured}$ PSF was strongly peaked and due to the large pixel of TRoPIC we could set just an upper limit to the angular resolution around 3.1 arcsec (Fig. 13C). The expected HEW with the parallel illumination produced by the Fresnel plate was 2.9 arcsec (Fig. 13D). A better sampling of the PSF can be obtained with PIXI camera, which has higher spatial resolution than TRoPIC despite a higher background level. The results shown in Fig. 14 confirm the data obtained with TRoPIC in the parallel beam configuration.
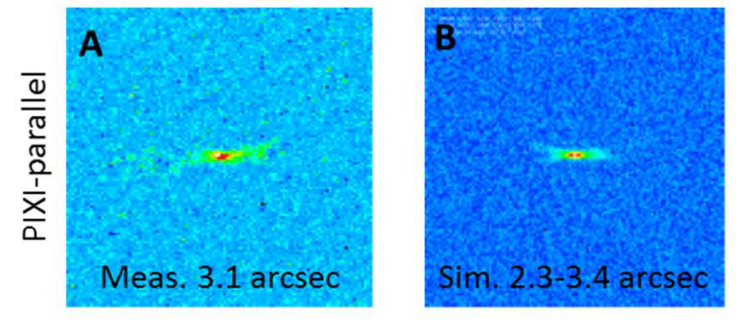

Figure 14. A. Best focus measured with PIXI sensor camera in parallel beam setup at $1.49 \mathrm{keV}, \mathrm{HEW}=3.1$ arcsec. B. Simulated best focus PSF. The uncertainty in the HEW value comes from the variable level of background assumed in the simulation.

\subsection{Coated mirror}

The mirror was coated at DTU Space by DC magnetron sputtering. Fig. 15A shows a picture of the DTU coating facility and Fig 15B shows the paraboloidal mirror after coating mounted inside the chamber. The bilayer Cr (thickness $=4.6 \mathrm{~nm}$ ) and $\mathrm{Pt}$ (thickness $=30 \mathrm{~nm}$ ) was selected by a characterization campaign performed on super-polished (roughness RMS < $2 \AA$ ) fused silica substrates [18]. The BEaTriX mirror after coating is shown mounted in the PANTER chamber in Fig. 15C. 

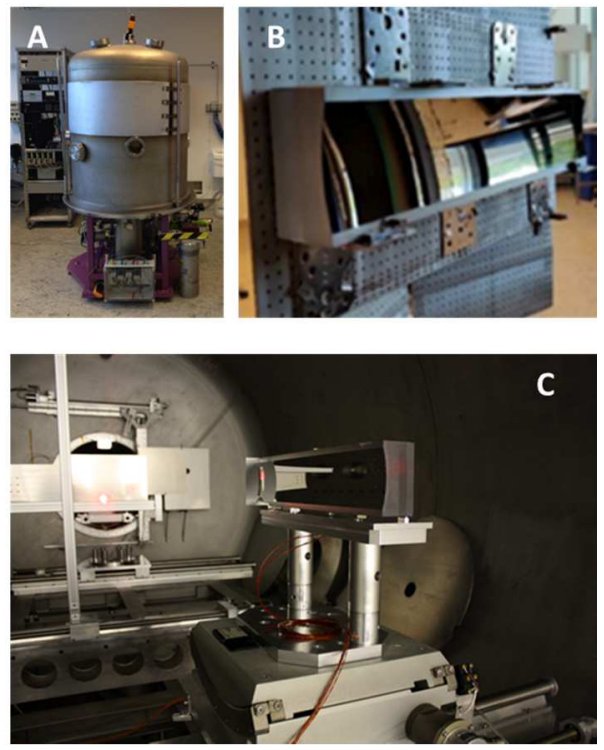

Figure 15. A. DC magnetron sputtering chamber at DTU Space. B. Coated mirror mounted in the sputtering vacuum chamber. C. Coated mirror mounted and aligned in the PANTER vacuum chamber.

The angular resolution measurements at $1.49 \mathrm{keV}$ were repeated on the mirror after coating, and some images of relevant results are reported in Fig. 16. Panel A (C) shows the best focus PSF measured with TRoPIC (PIXI) camera in divergent (parallel) beam configuration. Both measurements are in very good agreement with the corresponding ones taken before coating (see Fig. 13A and 14A resp.), within the measurement uncertainty, which are also displayed in Fig. 16 panel B and D for the reader's convenience. These results indicate no performance degradation due to the deposited coating at this low X-ray energy.
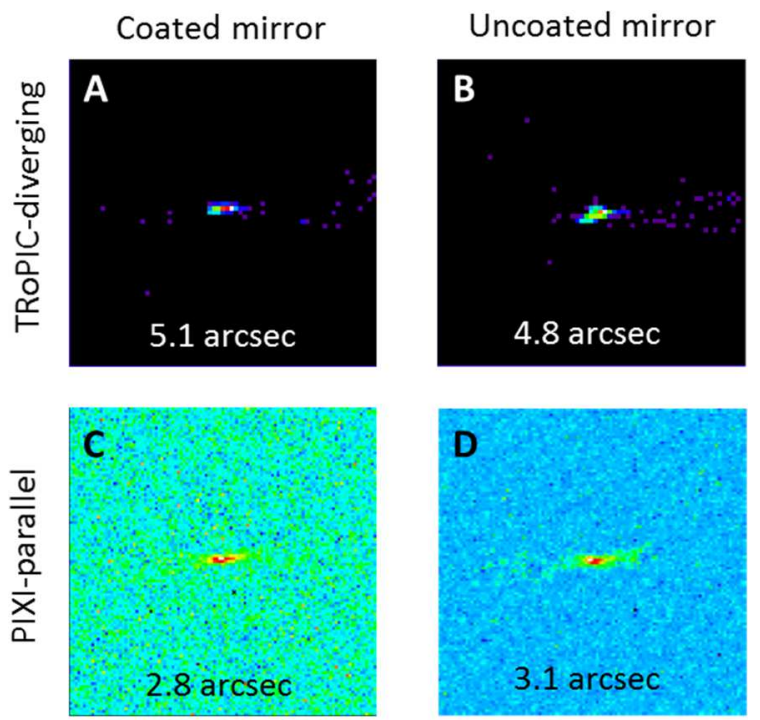

Figure 16. A (B). Best focus measured with TRoPIC in diverging beam setup on the coated (uncoated) mirror. HEW $=5.1$ $\operatorname{arcsec}(4.8 \operatorname{arcsec})$. C (D). Best focus measured with PIXI in parallel beam setup on the coated (uncoated) mirror. HEW = $2.8 \operatorname{arcsec}(3.1 \operatorname{arcsec})$. Measurements were made at $1.49 \mathrm{keV}$.

The presence of the reflective coating enabled to test the mirror performance also at $4.51 \mathrm{keV}$ energy. However, this was feasible only in the configuration with diverging beam, because the collimating zone plate does not work at this energy. The angular resolution with the divergent beam was measured 6.5 arcsec HEW, to be compared with the corresponding 5.1 arcsec HEW measured at $1.49 \mathrm{keV}$ (Fig. 17). There is some evidence of HEW degradation due to scattering. In order to clarify the origin of scattering we characterized the roughness of the coated surface with CCI 
(10X and 50X) measurements at three positions. We found that the roughness values slightly decreased after coating. This observation seems to confirm that the increase of scattering is not due to the coating deposition. Instead, and most probably, this scattering would originate from the part of the surface illuminated at PANTER which is outside the moon-shaped area (red part in Fig. 4C), which represents the area of the paraboloid involved in the generation of the expanded beam. From previous CCI measurement sessions on the uncoated glass surface, we knew that the sampled points displaying the highest roughness were outside the moon-shaped area and mostly located near the edge of the optical area.
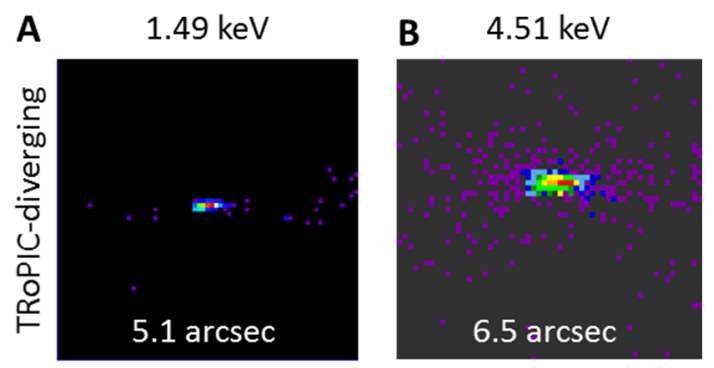

Figure $17 \mathrm{~A}(\mathrm{~B})$. Best focus measured with TRoPIC in diverging beam setup on the coated mirror at $1.49 \mathrm{keV}(4.51 \mathrm{keV})$ energy. HEW = $5.1 \operatorname{arcsec}(6.5 \operatorname{arcsec})$.

We estimated that the difference of $\sim 1.5$ arcsec between images in Fig. 17 would probably yield 4.5 arcsec in parallel beam setup at $4.51 \mathrm{keV}$. Such an angular resolution for the mirror, in turn, would contribute to the vertical and horizontal collimation of the output BEaTriX expanded beam by about 1.0 and 2.5 arcsec HEW respectively, according to the simulations presented in Fig. $18[19,20]$. From the considerations above on the possible origin of the increased scattering at $4.51 \mathrm{keV}$, we expect these results may represent an upper limit to the foreseen performance of BEaTriX.

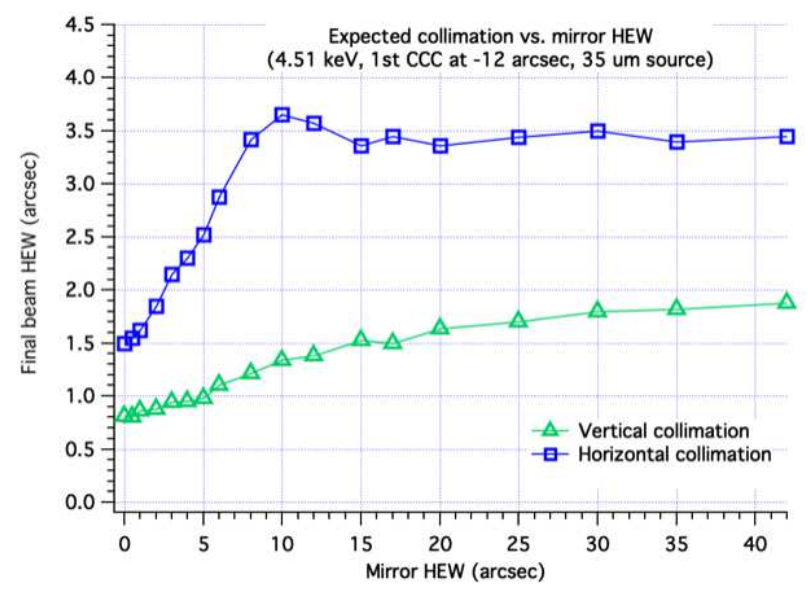

Figure 18. Simulation of the expected vertical and horizontal collimation of the BEaTriX output beam at $4.51 \mathrm{keV}$ as a function of the mirror quality.

\section{CONCLUSIONS}

In this paper, we presented the manufacturing and testing of the paraboloidal mirror designed to collimate the X-ray beam of the BEaTriX facility under construction now at INAF-Brera Astronomical Observatory. BEaTriX was conceived as a new type of beamline to support the development of the ATHENA X-ray telescope, as it is designed for the acceptance testing and calibration of the SPO mirror modules at fast rate. The mirror was designed to ensure collimation and uniformity to the BEaTriX beam. Bonnet polishing, pitch smoothing and IBF techniques were employed at INAF-Brera Astronomical Observatory for the mirror manufacturing. We presented metrology results after 
these processes, assessing the quality achieved by the mirror surface. We presented best focus PSF results of the X-ray test campaign conducted at PANTER before coating. A Cr-Pt two-layer coating was designed and made by magnetron sputtering at DTU Space. X-ray PANTER test campaign repeated on the Cr-Pt coated mirror confirmed the results on the uncoated glass mirror at low energy $(1.49 \mathrm{keV})$. In particular, the angular resolution at the best focus position was measured close to 3 arcsec HEW using a parallel beam setup enabled by a Fresnel zone plate. This performance of the mirror at $1.49 \mathrm{keV}$ matches the requirement of 3 arcsec HEW originally set for the surface quality. Measurements performed with diverging beam at $4.51 \mathrm{keV}$ displayed a degradation of the HEW value. This was attributed to an increase of scattering most probably originated in the part of the surface outside to that truly involved in the generation of the expanded beam. From the measurements performed at $4.51 \mathrm{keV}$, we inferred a 4.0-4.5 arcsec HEW in parallel beam setup. By this estimated value, the contribution of the mirror quality to the performance of the BEaTriX output beam foresees a vertical and horizontal collimation close to 1.0 and $2.5 \operatorname{arcsec} \mathrm{HEW}$, respectively. These values, to the best of our actual knowledge, may represent a conservative estimate and they will be verified in the coming months, after beam alignment, by qualifying the beam collimation with an ad hoc fabricated Hartmann plate.

\section{ACKNOWLEDGMENTS}

The project is financed by ESA (contract \# 4000123152/18/NL/BW), AHEAD (grant \#654215 and \#871158), ASI (grant \# 2019-27-HH.0) and INAF funds. We thank M. Rossi and L. Arcangeli (Media Lario S.r.l.) for technical support.

\section{REFERENCES}

[1] Nandra, K., Barret, D., Barcons, X., et al., "The Hot and Energetic Universe: A White Paper presenting the science theme motivating the Athena mission," http://arxiv.org/abs/1306.2307 (2013).

[2] Collon, M. J., Babic, L., Barrière, N. M., Bayerle, A., Castiglione, L., et al., "X-ray mirror development and production for the Athena telescope," Proc. SPIE 11852, 118521Z (2021).

[3] Bavdaz, M., Wille, E., Ayre, M., Ferreira, I., Shortt, B., et al., "The Athena X-ray optics development and accommodation," Proc. SPIE 11852, 1185220 (2021).

[4] Handick, E., Cibik, L., Krumrey, M., Müller, P., Barrière, N. M., et al., "Upgrade of the X-ray parallel beam facility XPBF 2.0 for characterization of silicon pore optics," Proc. SPIE 11444, 114444G (2020).

[5] Vacanti, G., Collon, M. J., Barrière, N. M., Landgraf, B., Günther, R., et al., "X-ray testing of Silicon Pore Optics," Proc. SPIE 11119, 111190I (2019).

[6] Bradshaw, M., Burwitz, V., Hartner, G., Langmeier, A., Vacanti, G., et al., "Testing ATHENA optics: a new measurement standard at the PANTER X-ray test facility," Proc. SPIE 11852, 1185223 (2021).

[7] Heinis, D., Carballedo, A., Colldelram, C., Cuní, G., Valls Vidal, N., et al., "X-ray facility for the characterization of the Athena mirror modules at the ALBA synchrotron," Proc. SPIE 11852, 1185222 (2021).

[8] Salmaso, B., Basso. S., Cotroneo V., Ghigo, M., Pareschi, G., et al., "Building the BEaTriX facility for the ATHENA mirror modules X-ray testing," Proc. SPIE this conference.

[9] Spiga, D., Pareschi, G., Pelliciari, C., Salmaso, B., Tagliaferri, G., "Functional tests of modular elements of segmented optics for X-ray telescopes via an expanded beam facility,” Proc. SPIE 8443, 84435F (2012).

[10] Spiga, D., Pelliciari, C., Salmaso, B., Arcangeli, L., Bianucci, G., et al., "Design and advancement status of the Beam Expander Testing X-ray facility (BEaTriX),” Proc. SPIE 9963, 996304 (2016).

[11] Sanchez del Rio, M., Cerrina, F., "Asymmetrically cut crystals for synchrotron radiation monochromators," Review of Scientific Instruments 63, 936 (1992).

[12] Salmaso, B., Basso, S., Giro, E., Spiga, D., Sironi, G., et al., "BEaTriX, the Beam Expander Testing X-Ray facility for testing ATHENA's SPO modules: progress in the realization," Proc. SPIE 11119, 111190N (2019).

[13] Vecchi, G., Salmaso, B., Basso, S., Sironi, G., Ghigo, M., et al., "BEaTriX, the Beam Expander Testing X-ray facility for testing ATHENA's SPO modules: the collimating mirror," Proc. SPIE 11119, 111191J (2019).

[14] Raimondi, L., Spiga, D., "Mirrors for X-ray telescopes: Fresnel diffraction-based computation of point spread functions from metrology," A\&A 573, A22 (2015).

[15] Ghigo, M., Vecchi, G., Basso, S., Citterio, O., Civitani, M., et al., "Ion figuring of large prototype mirror segments for the E-ELT", Proc. SPIE 9151, 91510Q (2014). 
[16] Sironi, G., Citterio, O., Pareschi, G., Negri, B., Ritucci, A., et al., "MPR: innovative 3D free-form optics profilometer," Proc. SPIE 8147, 814718 (2011).

[17]Menz, B., Brauninger, H., Burwitz, V., Hartner, G., Predehl, P., "Studying ATHENA optics with divergent and collimated X-ray beams,” Proc. SPIE 9144, 91445J (2014).

[18] Spiga, D., Salmaso, B., Vecchi, G., "Coating samples for the BEaTriX mirrors: surface roughness analysis," INAF$\mathrm{OAB}$ internal report $01 / 2021$.

[19] Spiga, D., Salmaso, B., Bavdaz M., Pelliciari C., Basso, S., et al., "Optical simulations for the laboratory-based expanded and collimated X-ray beam facility BEaTriX,” Proc. SPIE 11110, 111100E (2019).

[20] Spiga D., Salmaso, B., Ghigo, M., Basso, S., Bavdaz, M., et al., "Performance simulations for the ground-based, expanded-beam X-ray source BEaTriX," Proc. SPIE this conference. 